\title{
Long Term Outcome of In-Stent Stenosis after Stent Assisted Coil Embolization for Cerebral Aneurysm
}

\author{
Sung Jin Kim, Young-Joon Kim, Jung Ho Ko \\ Department of Neurosurgery, Dankook University College of Medicine, Cheonan, Korea
}

Objective : The objective of this study was to evaluatelong-term radiologic prognosis and characteristics of in-stent stenosis (ISS) after stent assisted coiling (SAC) for cerebral aneurysm and analyze its risk factors.

Methods : Radiological records of 362 cases of SAC during 10 years were retrospectively reviewed. Patients were included in this study if they had follow-up angiogram using catheter selected angiography at least twice. All subjected were followed up from 12 months to over 30 months. Of 120 patients, 123 aneurysms were enrolled. Patient data including age, sex, aneurysm size, neck size, procedural complication, kinds of stent, ISS associated symptom, ruptured state, location of ISS, degree of ISS, radiologic prognosis of ISS, follow-up period of time, and medical comorbidities such as hypertension, diabetes mellitus (DM), dyslipidemia, and smoking were collected.Statistical comparisons of group clinical characteristics were conducted for the total population.

Results : Among 123 casesof aneurysm, 22 cases (17.9\%) of ISS were revealed on follow-up angiography. Multiple stenting was performed in three cases and intra-procedural rupture occurred in two cases. Most cases were asymptomatic and symptomatic stenosis was identified in only one case. Sixteen cases were ruptured aneurysm. Mild stenosis was observed in 11 cases. Moderate stenosis was found in eight cases and severe stenosis was identified in three cases. Mean timing of identification of ISS was 8.90 months. The most common type was proximal type. Most cases were improved or not changed on follow-up angiography. Only one case was aggravated from mild stenosis to occlusion of parent artery. Mean follow-up period was 44.3 months. We compared risk factors and characteristic between ISS group and non-ISS group using univariate analysis. Multiple stenting was performed for three cases (13.6\%) of the ISS group and four cases (4.0\%) of the non-ISS group, showing no statistical difference between the two groups $(p=0.108)$. Additionally, the proportion of patients who had more than two risk factors among four medical risk factors (hypertension, DM, dyslipidemia, and smoking) was higher in the ISS group than that in the non-ISS group, the difference between the two was not statistically significant either ( $31.8 \%$ vs. $12.9 \%, p=0.05)$.

Conclusion : Clinical course and long-term prognosis of ISS might be benign. Most cases of ISS could be improved or not aggravated. Control of medical co-morbidity might be important. To the best of our knowledge, our study had more cases with longer follow-up period of time than other reports.

Key Words : Intracranial aneurysm · Stents · Long term adverse effects · Risk factors.

\footnotetext{
- Received : April 4, 2019 •Revised : May 14, 2019 •Accepted : June 3, 2019

- Address for reprints : Young-Joon Kim

Department of Neurological Surgery, Dankook University, College of Medicine, 201 Manghyang-ro, Dongnam-gu, Cheonan 31116, Korea Tel : +82-41-550-3980, E-mail : kimyj@dankook.ac.kr, ORCID : http://orcid.org/0000-0003-0499-1852
}

This is an Open Access article distributed under the terms of the Creative Commons Attribution Non-Commercial License (http://creativecommons.org/licenses/by-nc/4.0) which permits unrestricted non-commercial use, distribution, and reproduction in any medium, provided the original work is properly cited. 


\section{INTRODUCTION}

Endovascular treatment for cerebral aneurysm has been remarkably developed. Stent assisted coiling (SAC) for complex aneurysm has been considered as the standard treatment. Intracranial stent for cerebral aneurysm is designed as a self-expanding type that can improve angiographic aneurysmal occlusion by serving as a scaffold to prevent coil prolapse. It can also enhance coil packing density and provide great stability to the coil mass while preventing recanalization ${ }^{7}$. However, in-stent stenosis (ISS) as delayed complication of this procedure has been reported. Some characteristics of them have been suggested by previous studies ${ }^{4,9,12,13,17,18,24,25)}$. These suggestions had limitations in that these studies had small numbers of cases with short-term follow up. In addition, most articles only considered high degree stenosis $(>50 \%)$ as meaningful ISS $^{9,12,13,18)}$. The pathophysiology of ISS is not well-known yet, although endothelial injury following stent deployment, allergy to metal stent components, ingrowth of endothelium, and intimal ingrowth have been considered as potentially putative factors $^{5}$. Additionally, we believe that medical factors such as metabolic syndrome, smoking, and other factors could be related to ISS formation. Thus, the objective of this study was to evaluate long-term radiologic prognosis and characteristics of ISS after SAC for cerebral aneurysm and analyze its risk factors.

\section{MATERIALS AND METHODS}

\section{Study population}

This retrospective study was approved by the Institutional Review Board of our hospital (2019-04-020). Due to the retrospective design of the study, consent was neither required by the IRB nor by the study team. Radiological records of 362 cases of SAC between March 2007 and December 2016 were retrospectively reviewed. Patients were included in this study if they had follow-up angiogram using catheter selected angiography at least twice. Patients were excluded if they were treated with a flow-diverter or they only had follow-up angiography within 12 months or no radiological follow-up. Of 120 patients, 123 aneurysms were enrolled. Patient data including age, sex, aneurysm size, neck size, procedural complication, kinds of stent, ISS associated symptom, ruptured state, location of ISS, degree of ISS, radiologic prognosis of ISS, follow-up period of time, and medical comorbidities such as hypertension, diabetes mellitus (DM), dyslipidemia, and smoking were collected.

\section{Procedure and medication}

We have performed stent assisted coil embolization when ratio of dome to neck was above 2 or it was difficult to make a stable frame by coiling alone. At the beginning of the procedure, a bolus of 5000 IU of heparin was administered after insertion of an introducer sheath followed by $1000 \mathrm{IU} /$ hour of heparin. The stent was deployed such that distal markers were placed more distally from the arising portion of neck and sac. Neuroform 2 or Neuroform 3 stent (Boston Scientific, Marlborough, MA, USA), Neuroform EZ (NF; Stryker, Cork, Ireland), and Enterprise (EP; Codman, West Chester, PA, USA) were used. Coil embolization was mainly performed with the introduction of a microcatheter through the cell of stent. Bare platinum coils used included Guglielmi detachable coil (Boston Scientific), Target coil (Stryker), and MicroPlex coil (MicroVention, Aliso Viejo, CA, USA). For unruptured cases, pretreatment with dual antiplatelet therapy (100 mg aspirin and $75 \mathrm{mg}$ clopidogrel per oral) was performed for five to seven days. Dual antiplatelet therapy (75 mg clopidogrel daily and $100 \mathrm{mg}$ aspirin daily indefinitely) was started one day later after the procedure was completed. For ruptured cases, instead of using dual antiplatelet therapy, intravenous aspirin lysine 500 to $900 \mathrm{mg}$ was injected immediately after the first coil deployment following stenting. Activated clotting time was maintained between 250 and 300 seconds as in usual cases. Dual antiplatelet therapy (same prescription as above) was started the next day after treatment.

\section{Follow-up protocol}

Our protocol of angiographic follow-up using only catheter selected angiography was described as follows. The first angiographic follow-up was conducted at 3 to 6 months postoperatively according to clinical outcome and immediate angiographic results. The next one was performed at 12 months later depending on angiographic results of the first follow-up. The third one was performed at 12 to 24 months later depending on results of the second follow-up. All subjected were followed up from 12 months to over 30 months. 


\section{Classifications and definitions}

We classified ISS according to its location in a stent into four types : proximal, middle, distal, and diffuse types. ISS was defined when vessel diameter around implanted stent was decreased in follow-up angiography compared to the original diameter on immediate post-stenting angiography. Degrees of ISS were classified as mild stenosis (below 30\% narrowing of parent artery), moderate (30\% to 50\%), and severe (above 50\% narrowing). Statistical comparisons of group clinical characteristics were conducted for the total population. Multiple medical risk factors were defined when patient had more than two risk factors among four medical risk factors (hypertension, DM, dyslipidemia, and smoking).

\section{Statistical analysis}

Univariate analysis was used to identify factors affecting ISS. Chi-square test or Fisher's exact test was used to analyze categorical variables while independent t-test was used for continuous variables. All statistical analyses were performed using SPSS version 23.0.0. (IBM, Armonk, NY, USA) Statistical significance was accepted at $p<0.05$.

Table 1. Demographic characteristics of all subjected $(n=123)$

\begin{tabular}{lc}
\hline & Value \\
\hline Mean age (years) & 51.69 \\
Female & $88(71.5)$ \\
Mean size (mm) & 6.03 \\
Mean neck (mm) & 3.55 \\
Multiple stenting & $7(5.7)$ \\
Procedural complication & $10(8.1)$ \\
Ruptured state & $84(68.3)$ \\
Pre-medication & $56(45.5)$ \\
HTN & $63(51.2)$ \\
DM & $10(8.1)$ \\
Dyslipidemia & $8(6.5)$ \\
Smoking & $16(13.0)$ \\
Multiple medical factors & $20(16.3)$ \\
Mean last follow-up (months) & 54.14 \\
\hline
\end{tabular}

$\overline{\text { Values are presented as number (\%). HTN : hypertension, DM : diabetes }}$ mellitus

\section{RESULTS}

Demographic characteristics of all subjected used in this study are summarized in Table 1. Their mean age was 51.69 years (range, 30 to 80 years). Of 120 patients, 32 were men and 88 were women. Of 123 aneurysm, mean aneurysm size was $6.0 \mathrm{~mm}$ (range, 2.1 to $28.3 \mathrm{~mm}$ ) and the mean size of the aneurysm neck was $3.6 \mathrm{~mm}$ (range, 1.1 to $9.2 \mathrm{~mm}$ ). Multiple stenting was performed for seven cases (5.7\%). Procedural complication such as intra-procedural rupture or thromboembolic event occurred in 10 cases (8.1\%). Ruptured aneurysms occurred in 84 cases $(68.3 \%)$, including 56 cases $(45.5 \%)$ premedicated with dual anti-platelet. Sixty-three patients $(52.5 \%)$ had hypertension. Ten (8.3\%) had DM and eight (66.7\%) had dyslipidemia. Sixteen patients (13.3\%) continued to smoke cigarette after treatment. Twenty patients (16.7\%) had multiple medical factors. The mean follow-up period was 54.14 months (range, 12 to 141 months).

Among 123 cases of aneurysm, 22 cases (17.9\%) of ISS were revealed on follow-up angiography. Radiological and clinical characteristics of 22 ISS cases are shown in Tables 2 and 3. There were six men and 16 women with a mean age of 52.7 years (range, 37 to 71 years). The most common locations of aneurysm were anterior communicating artery (AcomA, six cases) and bifurcation of middle cerebral artery (MCA, six cases). Multiple stenting was performed in three cases (13.6\%) and intra-procedural rupture occurred in two cases $(9.1 \%)$. Most cases were asymptomatic and symptomatic stenosis (transient ischemic attack) was identified in only one case. Sixteen cases $(68.3 \%)$ were ruptured aneurysm. Mild stenosis was observed in 11 cases (50.0\%). Moderate stenosis was found in eight cases $(36.4 \%)$ and severe stenosis was identified in three cases $(13.6 \%)$. Mean timing of identification of ISS was 8.90 months (range, 6 to 17 months). The most common type was proximal type (10 cases, $45.5 \%)$. Most cases were improved (12 cases, $54.5 \%$ ) or not changed (nine cases, $40.9 \%$ ) on follow-up angiography. Only one case was aggravated from mild stenosis to occlusion of parent artery (Case 15, Fig. 1). Mean follow-up period was 44.3 months (range, 18 to 95 months).

We compared risk factors and characteristic between ISS group and non-ISS group using univariate analysis (Table 3). Proportion of ISS in small vessels such as AcomA and bifurcation of MCA was greater than that of others, however, there 
Long Term Outcome of In-Stent Stenosis | Kim SJ, et al.

Table 2. Radiological and clinical characteristics of 22 ISS cases

\begin{tabular}{|c|c|c|c|c|c|c|c|c|c|c|c|c|c|}
\hline No & Age & Sex & Location & $\begin{array}{c}\text { Kinds of } \\
\text { stent }\end{array}$ & $\begin{array}{l}\text { Complica- } \\
\text { tion }\end{array}$ & Sx. & Ruptured & Degree & Type & Timing & prognosis & $\begin{array}{c}\text { Final } \\
f / u\end{array}$ & Past Hx \\
\hline 1 & 49 & M & AcomA & Neu3 & 0 & No & Ruptured & Mild & Diffuse & 12 & Improved & 24 & HTN \\
\hline 2 & 37 & M & AcomA & Enter & 0 & No & Ruptured & Severe & Distal & 12 & Not changed & 23 & HTN, smoking \\
\hline 3 & 57 & $\mathrm{~F}$ & AcomA & Neu3 & 0 & No & Ruptured & Moderate & Distal & 6 & Not changed & 30 & No \\
\hline 4 & 52 & $\mathrm{~F}$ & AcomA & NeuEz & 0 & No & Ruptured & Mild & Distal & 6 & Improved & 34 & No \\
\hline 5 & 41 & $\mathrm{~F}$ & AcomA & NeuEz & $\mathrm{R}$ & No & Ruptured & Moderate & Proximal & 6 & Improved & 38 & HTN \\
\hline 6 & 57 & $\mathrm{~F}$ & AcomA & NeuEz & 0 & No & Ruptured & Mild & Distal & 6 & Not changed & 30 & HTN \\
\hline 7 & 58 & M & PcomA & Neu3 & 0 & No & Ruptured & Moderate & Proximal & 17 & Not changed & 31 & DM, HTN \\
\hline 8 & 56 & $\mathrm{~F}$ & Opthal. & Neu3 & 0 & No & Unruptured & Mild & Proximal & 6 & Not changed & 53 & HTN \\
\hline 9 & 59 & F & PcomA & Enter & 0 & No & Unruptured & Moderate & Distal & 13 & Not changed & 63 & HTN \\
\hline 10 & 51 & $\mathrm{~F}$ & Opthal. & NeuroformEz & 0 & No & Ruptured & Mild & Distal & 6 & Not changed & 42 & HTN \\
\hline 11 & 70 & $F$ & PcomA & NeuroformEz & 0 & No & Ruptured & Mild & Middle & 15 & Improved & 40 & HTN, smoking \\
\hline 12 & 49 & M & MCAbif & Neu3+Neu3 & $\mathrm{R}$ & No & Ruptured & Moderate & Proximal & 12 & Not changed & 65 & HTN \\
\hline 13 & 71 & $\mathrm{~F}$ & MCAbif & Neu3 & 0 & No & Unruptured & Mild & Diffuse & 12 & Improved & 65 & $\begin{array}{c}\text { DM, HTN, } \\
\text { dyslipidemia }\end{array}$ \\
\hline 14 & 48 & M & MCAbif & Neu3 & 0 & No & Ruptured & Moderate & Proximal & 12 & Improved & 37 & No \\
\hline 15 & 49 & $\mathrm{~F}$ & MCAbif & Neu3 & 0 & No & Ruptured & Mild & Proximal & 6 & Aggravated & 65 & DM, HTN, smoking \\
\hline 16 & 52 & $\mathrm{~F}$ & MCAbif & Neu3+Enter & 0 & No & Ruptured & Severe & Proximal & 6 & Improved & 18 & HTN \\
\hline 17 & 56 & $\mathrm{~F}$ & MCAbif & Neu3 & 0 & TIA & Unruptured & Severe & Proximal & 6 & Improved & 28 & Dyslipidemia, HTN \\
\hline 18 & 66 & $\mathrm{~F}$ & SCA & Neu3 & 0 & No & Ruptured & Mild & Distal & 6 & Not changed & 95 & HTN \\
\hline 19 & 47 & $\mathrm{~F}$ & PICA & Neu3 & 0 & No & Ruptured & Moderate & Distal & 13 & Improved & 38 & No \\
\hline 20 & 41 & M & BT & Neu3 & 0 & No & Unruptured & Mild & Proximal & 6 & Improved & 86 & DM, smoking \\
\hline 21 & 50 & $\mathrm{~F}$ & VA & Neu3+NeuEz & 0 & No & Unruptured & Mild & Proximal & 6 & Improved & 32 & HTN \\
\hline 22 & 44 & $F$ & VA & Neu3 & 0 & No & Ruptured & Moderate & Middle & 6 & Improved & 37 & No \\
\hline
\end{tabular}

ISS : in-stent stenosis, Sx. : symptom, f/u : follow-up, Hx : medical history, M : male, AcomA : anterior communicating artery, Neu3 : Neuroform3, HTN : hypertension, Enter : Enterprise, F : female, NeuEz : NeuroformEz, R : procedural rupture, PcomA : posterior communicating artery, Opthal. : opthalmic artery, MCAbif : bifurcation of Middle cerebral artery, SCA : stent assisted coiling, PICA : posterior inferior cerebral artery, BT : basilar top, VA : vertebral artery

Table 3. Comparison of risk factors and characteristic between ISS group and non-ISS group

\begin{tabular}{lccc}
\hline & ISS (n=22) & Non-ISS (n=101) & p-value \\
\hline Age $>55$ years & $9(40.9)$ & $39(38.6)$ & 1.000 \\
\hline Female & $16(72.7)$ & $72(71.3)$ & 1.000 \\
\hline AcomA \& MCAbif aneurysm & $12(54.5)$ & $42(41.6)$ & 0.191 \\
Multiple stenting & $3(13.6)$ & $4(4.0)$ & 0.108 \\
\hline Procedural complication & $2(9.1)$ & $8(7.9)$ & 1.000 \\
Ruptured state & $16(72.7)$ & $68(67.3)$ & 0.801 \\
\hline Pre-medication & $9(40.9)$ & $47(46.5)$ & 0.101 \\
HTN & $16(72.7)$ & $47(46.5)$ & 0.101 \\
DM & $4(18.2)$ & $6(5.9)$ & 0.078 \\
Dyslipidemia & $2(9.1)$ & $6(5.9)$ & 0.633 \\
Smoking & $4(18.2)$ & $12(11.9)$ & 0.484 \\
Multiple medical factors & $7(31.8)$ & $13(12.9)$ & 0.05 \\
\hline
\end{tabular}

Values are presented as number (\%). ISS : in-stent stenosis, AcomA : anterior communicating artery, MCAbif : middle cerebral artery bifurcation, HTN : hypertension, DM : diabetes mellitus 
were no statistical significance $(54.5 \%$ vs. $41.6 \% p=0.191)$. Multiple stenting was performed for three cases (13.6\%) of the ISS group and four cases $(4.0 \%)$ of the non-ISS group, showing no statistical difference between the two groups. Proportions of procedural complication, ruptured state, and premedication of dual antiplatelet before treatment were also similar between the two groups. Although the proportion of patients who had a history of hypertension, DM, dyslipidemia, or smoking in the ISS group was higher than that in the nonISS group, the difference between the two groups was not statistically significant. Additionally, the proportion of patients who had more than two risk factors among four medical risk factors (hypertension, DM, dyslipidemia, and smoking) was higher in the ISS group than that in the non-ISS group, the difference between the two was not statistically significant either $(31.8 \%$ vs. $12.9 \%, p=0.05)$.

\section{Case illustration}

\section{Case 15}

A 49-year-old female had a ruptured aneurysm and experienced a severe headache. Patient had history of hypertension, DM, smoking, and stenting for coronary artery stenosis. A saccular aneurysm on the left bifurcation of MCA was treated by stent-assisted coil embolization with Neuroform 3 stent. Immediate post-embolization angiography showed nearly completely occluded aneurysm without any lesion around the stent (Fig. 1A). However, mild ISS in the proximal portion of
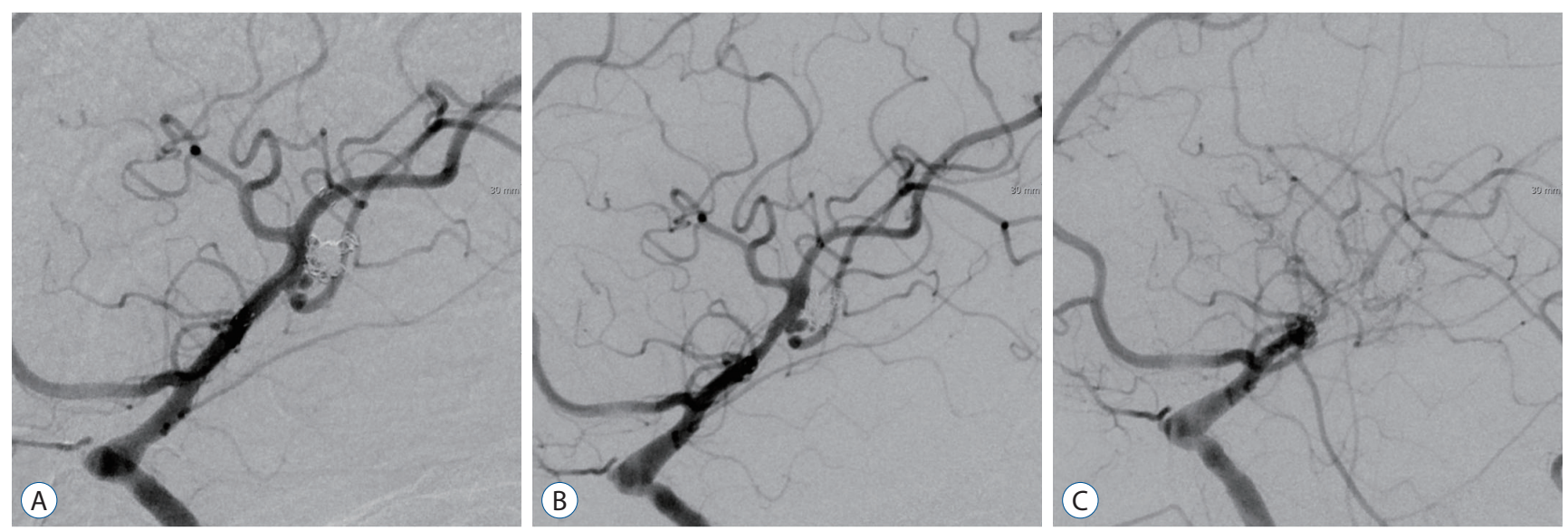

Fig. 1. Aggravated case of ISS (Case 15). A : Immediate post-embolization angiography shows nearly completely occluded aneurysm without any lesion around the stent. B: Mild ISS in the proximal portion of stent is revealed on follow-up angiography 6 months later. C: Post-embolization 12-month angiography shows an occlusion of middle cerebral artery from the portion of ISS. ISS : in-stent stenosis.
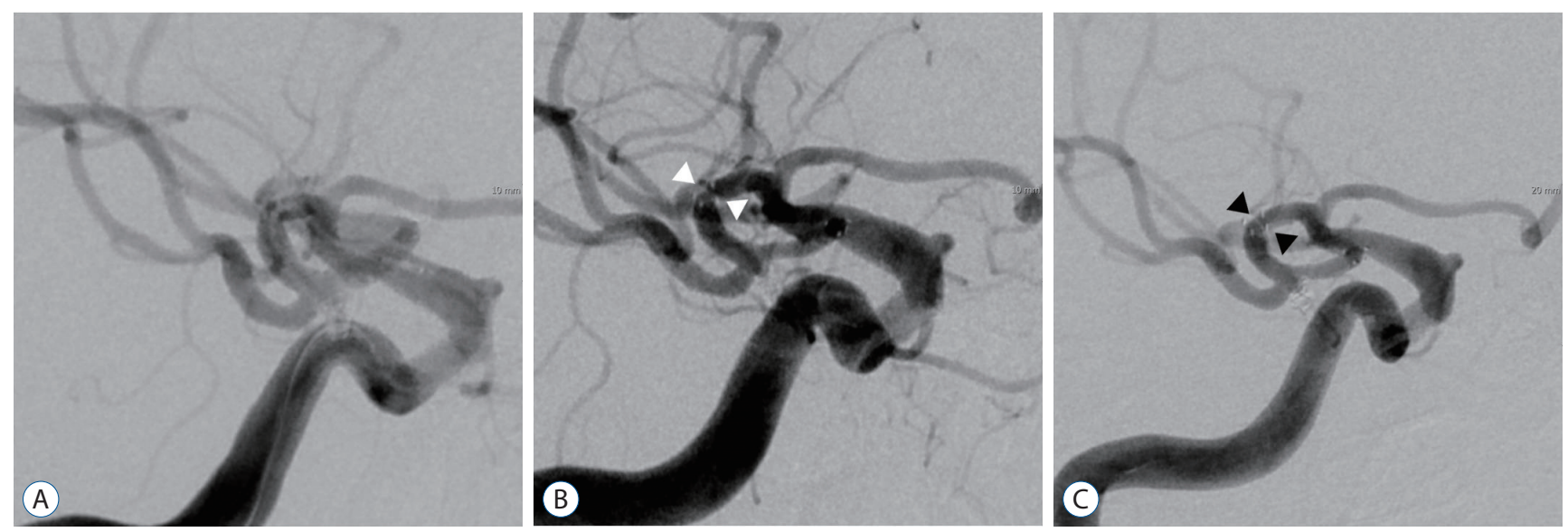

Fig. 2. Improved cases of ISS (Case 17). A: Well-occluded aneurysm without in-stent complication is seen on immediate post-embolization angiography. B : Angiography shows severe ISS ( $>50 \%$, white arrowheads) in the proximal portion of the implanted stent. C: A recovery from severe to mild stenosis (black arrowheads) is identified on follow-up angiography at 28 months post embolization. ISS : in-stent stenosis. 
stent was revealed on follow-up angiography 6 months later (Fig. 1B). Post-embolization 12-month angiography showed an occlusion of MCA from the portion of ISS (Fig. 1C). She was unable to quit smoking at that time. Final angiography was performed at post-embolization 65 months and angiographic finding was not changed. Fortunately, she had no symptom associated with the obstruction of MCA. Her clinical course has been uneventful during the period of follow-up.

\section{Case 17}

A 56-year-old female patient had incidental right bifurcation of MCA aneurysm. She had medical histories of hypertension and dyslipidemia. Stent-assisted coil embolization using Neuroform 3 stent was performed for the aneurysm. The stent was deployed from M1 to M2. Well-occluded aneurysm without in-stent complication was seen on immediate postembolization angiography (Fig. 2A). The patient experienced transient weakness of the left arm and dysarthria 6 months later. Angiography showed severe ISS ( $>50 \%)$ in the proximal portion of the implanted stent (Fig. 2B). After Plavix and Atorvastatin were added into her prescription, a recovery from severe to mild stenosis was identified on follow-up angiography at 28 months post embolization (Fig. 2C). She have not experience transient ischemic symptom again.

\section{DISCUSSION}

Table 4 shows characteristics and follow-up data of ISS after stenting with Neuroform or Enterprise stent according to published literatures. They have some differences depending on ISS rate, kinds of follow-up, kinds of stents, and criteria of ISS. Incidence rates of ISS and symptomatic stenosis after SAC have been reported to be $2.3-7.8 \%$ and $0-1.3 \%$, respectively ${ }^{4,9,12,18,22,25)}$. According to literatures, ISS rates after Neuroform stenting are relatively low, ranging from $0 \%$ to $6.3 \%^{1,4,8,17,24)}$. ISS rate in our study was revealed to be $17.9 \%$, which was relatively higher than rate in other studies. Significant ISS was defined as above $50 \%$ stenosis in most studies, ${ }^{9,12,13,18}$ or classified as 33.3\% and $66.6 \%$ stenosis in some studies ${ }^{5,7)}$. Considering that our criterion of ISS was below $30 \%$ stenosis, our incidence $(17.9 \%$ ) would be reasonable. Additionally, Case 15 (Fig. 1) showed that even mild ISS (below 30\%) stenosis could be aggravated to occlusion. Thus, mild or moderate ISS could be clinically significant. In another study with similar criterion ${ }^{5}$, the ISS rate after LVIS stent-assisted coiling was $86.7 \%$. This result showed that the ISS rate could be variable depending on kinds of stent because greater surface area coverage and smaller cell-size of stents could be risk factors of ISS $S^{3,5,20)}$.

Spontaneous resolution of the stenosis has also been observed in several case reports with a rate of $12.5-66.6 \% \%^{9,13,18,25)}$. Some authors have suggested that aneurysmal stenting seems to have a more benign course than atherosclerosis based on

Table 4. Characteristics and follow-up data of ISS after stenting in literatures

\begin{tabular}{|c|c|c|c|c|c|c|c|c|}
\hline No. & Study & $\begin{array}{l}\text { Kinds of } \\
\text { follow-up }\end{array}$ & Kinds of stent & Criteria of ISS & ISS rate & Symptom & Progress & $\begin{array}{c}\text { Follow-up } \\
\text { period of time }\end{array}$ \\
\hline 1 & Biondi et al. ${ }^{4)}(2007)$ & DSA & Neuroform & No data & $1 / 42(2.4 \%)$ & Asymtomatic & No data & Mean 9 month \\
\hline 2 & Fiorella et al." (2006) & MRA, DSA & Neuroform & $>50 \%$ & 9/161 (5.6\%) & $\begin{array}{c}2 / 9 \\
\text { (symptomatic) }\end{array}$ & $1 / 9$ progress & 14 to 17 month \\
\hline 3 & Kanaan et al. ${ }^{12)}(2010)$ & DSA & $\begin{array}{l}\text { Neuroform, } \\
\text { Enterprise }\end{array}$ & $>50 \%$ & $5 / 133(3.8 \%)$ & $\begin{array}{c}2 / 5 \\
\text { (asymptomatic) }\end{array}$ & No data & $\begin{array}{l}\text { Mean } 15.4 \\
\text { month }\end{array}$ \\
\hline 4 & Kulcsár et al. ${ }^{13)}(2013)$ & MRA, DSA & Neuroform & $>50 \%$ & $3 / 117(2.6 \%)$ & Asymtomatic & $2 / 3$ improved & Mean 33 month \\
\hline 5 & Maldonado et al. ${ }^{17)}$ (2011) & DSA, MRA & Neuroform & No data & $3 / 76(3.9 \%)$ & Asymtomatic & No data & $\begin{array}{c}\text { Mean } 25.7 \\
\text { month }\end{array}$ \\
\hline 6 & Mocco et al. ${ }^{18)}(2011)$ & DSA & Enterprise & $>50 \%$ & $3 / 110(2.7 \%)$ & Asymtomatic & $1 / 3$ improved & Mean 175 days \\
\hline 7 & Vendrell et al. ${ }^{24)}$ (2011) & MRA, DSA & $\begin{array}{l}\text { Neuroform, } \\
\text { Enterprise }\end{array}$ & No data & $2 / 47(4.3 \%)$ & Asymtomatic & No data & No data \\
\hline 8 & Yoon and $\mathrm{Kim}^{25)}$ (2010) & DSA & $\begin{array}{l}\text { Neuroform, } \\
\text { Enterprise }\end{array}$ & $>20 \%$ & $8 / 102(7.8 \%)$ & Asymtomatic & 1/8 improved & No data \\
\hline
\end{tabular}

ISS : in-stent stenosis, DSA : digital subtraction angiography, MRA : magnetic resolution angiography 
the rate of adverse events and spontaneous resolution ${ }^{11,15,16)}$. In addition, Gao et al. ${ }^{10)}$ have suggested that ISS might be dynamic and spontaneously resolvable. They presented evidence indicating that stenosis peaked at 4-6 months following the procedure, but resolved at 12-24 months post-treatment. However, most studies had small number of cases (range, 1 to 9 cases) and short-term or mid-term follow-up period (range, 6 to 33 months). In addition, continuity of follow-up appeared to be lacking. Thus, we tried to identify a change in ISS from formation to final result during the follow-up period. Our mean follow-up period was 44.3 months, ranging from 18 to 95 months in 22 cases. We checked changes of ISS sequentially for a relatively long-term. Ultimately, we found that most cases were improved (12 cases, 54.5\%) or not changed (nine cases, $40.9 \%$ ) on follow-up angiography. Only one case was aggravated from mild stenosis to occlusion of parent artery. Although the prognosis of ISS is benign, physicians should focus on aggravation cases because all ISS cases have potential to aggravate. Whether asymptomatic stenosis could be changed to asymptomatic or symptomatic aggravation of stenosis in follow-up is unknown. Our results suggest that special attention and follow-up are needed for ISS.

In most studies, both magnetic resolution angiography (MRA) with time of flight (TOF) technique or contrast enhancement (CE) and catheter selected angiography are used to identify IIS through radiologic follow-up ${ }^{9,13,17,24)}$. Many endovascular surgeons have their own protocols of image followup after endovascular coiling for intracerebral aneurysm depending on their experience and situation of their institute. We did at least three times of follow-up cerebral angiography using digital subtraction angiography (DSA) for 3 to 4 years. Some physicians have suggested the usefulness of MRA using TOF technique or CE for follow-up ${ }^{14,23}$. Most physicians might have performed follow-up imaging using both techniques. Follow-up using DSA has been chosen as the only method in our institute because DSA could is still a gold standard and promising technique for cerebral disease, although Prabhakaran et al. ${ }^{21)}$ have suggested that MRA could be a promising screening test to detect intracranial in-stent stenosis. Because only one type of radiologic technique was used in our study, we were able to collect constant image and prove differences of angiographic change more accurately.

The degree of neo-intimal hyperplasia and ISS after SAC is probably linked to the severity of endothelial injury induced during stent deployment and further manipulations that can affect stent stability during the initial procedure ${ }^{13)}$. Recent multicenter studies focusing on aneurysm occlusion after Ystenting has reported that immediate total or near total occlusion is achieved in a high rate of cases ${ }^{5,6)}$. Multiple stenting is a very meticulous and high-skilled technique comparing with simple stenting. It can result in intimal injury easily. In our study, multiple stenting was performed in three cases (13.6\%) of the ISS group and four cases (4.0\%) in the non-ISS group. Although the rate of multiple stenting performed showed discrepancy between the two groups, the difference was not statistically significant. Larger samples size with prospective study design is needed to obtain a definite conclusion. SAH by itself could be a vulnerable condition to form ISS. The intracerebral hemodynamics might be unstable and patient might be in hypercoagulable state ${ }^{13)}$. Additionally, physician's understanding about vascular geometry around aneurysm for ruptured cases might be lower than that for unruptured cases because most ruptured cases were in emergent situation. However, there was no significant difference in SAH or premedication rate between ISS and non-ISS groups in our study. Although the tortuosity of vasculature was excluded from our study as a risk factor because it was difficult to quantify a tortuosity objectively, the tortuosity of vasculature might be a potential risk factor affecting intimal injury from stenting. In addition, medical condition and smoking might be risk factors related to repair mechanism. This is because metabolic syndrome such as hypertension, DM, dyslipidemia, and smoking are well-known risk factors of atherosclerosis and instent stenosis of stenting for atherosclerotic disease ${ }^{2,19)}$. However, we failed to prove a difference in medical risk factor ratio between ISS and non-ISS groups. Although the proportion of patients with multiple medical factor (over two factors among hypertension, DM, dyslipidemia, and smoking) did not show statistically significant difference between the two groups ( $p=0.05$ ), it showed a tendency to have relations with the occurrence of ISS. Multiple factors and complex mechanism could influence the formation of ISS. Our results suggest that it might be better to control medical co-morbidities and quit smoking for ISS prevention after treatment.

The present study has some limitations. First, it was performed using a retrospective chart review. This situation might have increased the risk of selection bias. Second, the number of patients enrolled in this study was too small to 
reach statistically significant conclusions. A future study with a larger number of patients is needed to establish risk factors of ISS. Despite these limitations, our study might provide guidance for planning treatment strategy when physicians encounter a similar situation.

\section{CONCLUSION}

Clinical course and long-term prognosis of ISS might be benign. Most cases of ISS could be improved or not aggravated. Control of medical co-morbidity might be important. To the best of our knowledge, our study had more cases with longer follow-up period of time than other reports.

\section{CONFLICTS OF INTEREST}

No potential conflict of interest relevant to this article was reported.

\section{INFORMED CONSENT}

Informed consent was obtained from all individual participants included in this study.

\section{AUTHOR CONTRIBUTIONS}

\author{
Conceptualization : YJK, JHK \\ Data curation : SJK \\ Formal analysis : SJK, YJK, JHK \\ Methodology : YJK, JHK \\ Project administration : YJK, JHK \\ Visualization: SJK \\ Writing - original draft : SJK, JHK \\ Writing - review \& editing : SJK, YJK, JHK
}

\section{References}

1. Akpek S, Arat A, Morsi H, Klucznick RP, Strother CM, Mawad ME : Selfexpandable stent-assisted coiling of wide-necked intracranial aneurysms: a single-center experience. AJNR Am J Neuroradiol 26 : 1223-1231,
2005

2. Banerjee C, Chimowitz MI : Stroke caused by atherosclerosis of the major intracranial arteries. Circ Res 120 : 502-513, 2017

3. Behme D, Weber A, Kowoll A, Berlis A, Burke TH, Weber W : Lowprofile Visualized Intraluminal Support device (LVIS Jr) as a novel tool in the treatment of wide-necked intracranial aneurysms: initial experience in 32 cases. J Neurointerv Surg $7: 281-285,2015$

4. Biondi A, Janardhan V, Katz JM, Salvaggio K, Riina HA, Gobin YP : Neuroform stent-assisted coil embolization of wide-neck intracranial aneurysms: strategies in stent deployment and midterm follow-up. Neurosurgery 61 : 460-468; discussion 468-469, 2007

5. Cho YD, Sohn CH, Kang HS, Kim JE, Cho WS, Hwang G, et al. : Coil embolization of intracranial saccular aneurysms using the Low-profile Visualized Intraluminal Support (LVISTM ${ }^{\text {TM}}$ ) device. Neuroradiology 56 : 543-551, 2014

6. Fargen KM, Mocco J, Neal D, Dewan MC, Reavey-Cantwell J, Woo HH, et al. : A multicenter study of stent-assisted coiling of cerebral aneurysms with a $Y$ configuration. Neurosurgery 73 : 466-472, 2013

7. Feng $X$, Qian Z, Liu P, Zhang B, Wang L, Guo E, et al. : Comparison of recanalization and in-stent stenosis between the low-profile visualized intraluminal support stent and enterprise stent-assisted coiling for 254 intracranial aneurysms. World Neurosurg 109 : e99-e104, 2018

8. Fiorella D, Albuquerque FC, Deshmukh VR, McDougall CG : Usefulness of the Neuroform stent for the treatment of cerebral aneurysms: results at initial (3-6-mo) follow-up. Neurosurgery 56 : 1191-1201; discussion 1201-1202, 2005

9. Fiorella D, Albuquerque FC, Woo H, Rasmussen PA, Masaryk TJ, MCDougall CG : Neuroform in-stent stenosis: incidence, natural history and treatment strategies. Neurosurgery $59:$ 34-42, 2006

10. Gao B, Safain MG, Malek AM : Enterprise stenting for intracranial aneurysm treatment induces dynamic and reversible age-dependent stenosis in cerebral arteries. J Neurointerv Surg 7 : 297-302, 2015

11. Hoit DA, Malek AM : Three-dimensional rotational angiographic detection of in-stent stenosis in wide-necked aneurysms treated with a selfexpanding intracranial stent. Neurosurgery 57 : 1228-1236; discussion 1228-1236, 2005

12. Kanaan H, Jankowitz B, Aleu A, Kostov D, Lin R, Lee K, et al. : In-stent thrombosis and stenosis after neck-remodeling device-assisted coil embolization of intracranial aneurysms. Neurosurgery 67 : 1523-1532; discussion 1532-1533, 2010

13. Kulcsár Z, Göricke SL, Gizewski ER, Schlamann M, Sure U, Sandalcioglu IE, et al. : Neuroform stent-assisted treatment of intracranial aneurysms: long-term follow-up study of aneurysm recurrence and in-stent stenosis rates. Neuroradiology $55:$ 459-465, 2013

14. Lane A, Vivian P, Coulthard A : Magnetic resonance angiography or digital subtraction catheter angiography for follow-up of coiled aneurysms: do we need both? J Med Imaging Radiat Oncol 59 : 163-169, 2015

15. Lubicz B, Collignon L, Raphaeli G, Pruvo JP, Bruneau M, De Witte O, et al. : Flow-diverter stent for the endovascular treatment of intracranial aneurysms: a prospective study in 29 patients with 34 aneurysms. Stroke 41 : 2247-2253, 2010 
16. Lylyk P, Miranda C, Ceratto R, Ferrario A, Scrivano E, Luna HR, et al. : Curative endovascular reconstruction of cerebral aneurysms with the pipeline embolization device: the Buenos Aires experience. Neurosurgery 64 : 632-642; discussion 642-643; quiz N6, 2009

17. Maldonado IL, Machi P, Costalat V, Mura T, Bonafé A : Neuroform stent-assisted coiling of unruptured intracranial aneurysms: short- and midterm results from a single-center experience with 68 patients. AJNR Am J Neuroradiol 32 : 131-136, 2011

18. Mocco J, Fargen KM, Albuquerque FC, Bendok BR, Boulos AS, Carpenter JS, et al. : Delayed thrombosis or stenosis following enterprise-assisted stent-coiling: is it safe? Midterm results of the interstate collaboration of enterprise stent coiling. Neurosurgery 69 : 908-913; discussion 913914,2011

19. Neeb ZP, Edwards JM, Alloosh M, Long X, Mokelke EA, Sturek M : Metabolic syndrome and coronary artery disease in Ossabaw compared with Yucatan swine. Comp Med 60 : 300-315, 2010

20. Poncyljusz W, Bilinski P, Safranow K, Baron J, Zbroszczyk M, Jaworski M, et al. : The LVIS/LVIS Jr. stents in the treatment of wide-neck intracranial aneurysms: multicentre registry. J Neurointerv Surg 7 : 524-529,
2015

21. Prabhakaran $S$, Warrior $L$, Wells KR, Jhaveri MD, Chen M, Lopes DK : The utility of quantitative magnetic resonance angiography in the assessment of intracranial in-stent stenosis. Stroke 40 : 991-993, 2009

22. Takemoto K, Tateshima S, Rastogi S, Gonzalez N, Jahan R, Duckwiler $G$, et al. : Disappearance of a small intracranial aneurysm as a result of vessel straightening and in-stent stenosis following use of an Enterprise vascular reconstruction device. J Neurointerv Surg 6 : e4, 2014

23. van Amerongen MJ, Boogaarts HD, de Vries J, Verbeek AL, Meijer FJ, Prokop $M$, et al. : MRA versus DSA for follow-up of coiled intracranial aneurysms: a meta-analysis. AJNR Am J Neuroradiol 35 : 1655-1661, 2014

24. Vendrell JF, Costalat V, Brunel H, Riquelme C, Bonafe A : Stent-assisted coiling of complex middle cerebral artery aneurysms: initial and midterm results. AJNR Am J Neuroradiol 32 : 259-263, 2011

25. Yoon KW, Kim YJ : In-stent stenosis of stent assisted endovascular treatment on intracranial complex aneurysms. J Korean Neurosurg Soc $48:$ 485-489, 2010 